\title{
ON CROSS CORRELATION BASED DISCRETE TIME DELAY ESTIMATION
}

\author{
Lei Zhang and Xiaolin Wu
}

\author{
Department of Electrical and Computer Engineering \\ McMaster University, Hamilton, Ontario, Canada, L8S 4K1
}

\begin{abstract}
Cross correlation function (CCF) is a powerful tool in time delay estimation and parabola functions are widely used as parametric models of it. However, no study has been done on the accuracy of the parabola approximation of CCF. In this paper we analyze the CCF of multi-sensors and derive the analytic forms of $\mathrm{CCF}$ for the stationary processes of exponential auto-correlation function and with respect to two important types of sensor kernels. We demonstrate that the Gaussian function is a better and more robust approximation of CCF than the parabola in these cases. This new approach leads to higher precision in time delay estimation using the CCF peak locating strategy.
\end{abstract}

\section{INTRODUCTION}

Multi-sensors are widely used for robust estimation, communication and data fusion. When multiple digital sensors of different physical characteristics and varying spatial locations sample a continuous-time signal, they produce correlated discrete-time sequences with some time displacements. The cross correlation function (CCF) is a powerful tool to register these discrete signals sampled by different sensors in time domain.

The time delay estimation of two analog signals through cross-correlation technique has been extensively studied [1-3] since Knapp and Carter gave a maximum likelihood estimator of the relative delay between two continuous signals [1]. However, this method needs to know the spectra of signals and noise and it applies to analog signals only. For digital systems a popular approach of time delay estimation is to locate the peak of the two discrete signals' CCF [4-6]. The delay is generally not an integral multiple of the sampling period. To estimate the delay in an arbitrary precision, a common technique is to fit the $\mathrm{CCF}$ by a parabola with three samples in the neighborhood of the peak correlation value.

In this paper we are interested in analyzing the CCF and the techniques to estimate the time delay by locating the CCF's peak position with a small number of measurements of the CCF. We will derive the analytical forms of the CCFs for Gaussian and Box sensor kernels for the class of stationary signals that have an exponentially decaying auto-correlation function (ACF). Our analysis will explain why the parabola function can fit the CCF reasonably well, as previously believed in the literatures. More importantly, we establish Gaussian function to be a better and more robust approximation of the CCF. Indeed, the new Gaussian model leads to superior performance in time delay estimation to the parabola-based method.

\section{PRELIMINARIES}

Consider a continuous information source $s(t)$ under observation. The discrete observation produced by any two sensors are $y_{i}(k)=x_{i}(k)+v_{i}(k), i=1,2$. Noise $v_{i}$ is zero mean and signal $x_{i}(k)=\int_{k-1) T+4_{i}}^{k T+4_{i}} s(t) g_{i}(t) d t$, where $T$ is the sampling period and $\Delta_{i}$ is the time delays of sensor i. $g_{i}(t)$ is the integral kernel of the sensor. Noises $v_{1}$ and $v_{2}$ are uncorrelated and also uncorrelated with $x_{i}$.

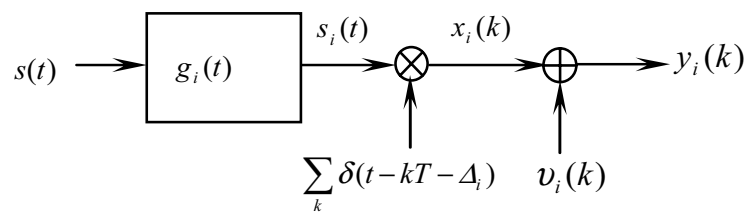

Figure 1. The sampling process of digital signal $y_{i}(k)$.

Fig. 1 shows the sampling procedure of $y_{i}(k)$. First $s_{i}(t)$ is generated by passing $s(t)$ through filter $g_{i}(-t)$, i.e., $s_{j}(t)=s(t) * g_{j}(-t)$. Then $s_{i}(t)$ is sampled by a Dirac sequence with period $T$ and time shift $\Delta_{i}$ so that

$$
x_{i}(k)=\int s_{i}(t) \cdot \delta\left(t-k T-\Delta_{i}\right) d t=s_{i}\left(k T-\Delta_{i}\right)
$$

Finally $y_{i}(k)$ is obtained by adding noise $v_{i}(k)$ to $x_{i}(k)$. Assume that $s(t)$ is a stationary process, then its ACF is $R_{s}(\tau)=R_{s}(-\tau)=E\{s(t) s(t+\tau)\}$. The CCF of signals $s_{1}(t)$ and $s_{2}(t)$ is given by [8]:

$$
R_{12}(\tau)=E\left\{s_{1}(t) s_{2}(t+\tau)\right\}=R_{s}(\tau) * g_{1}(-\tau) * g_{2}(\tau)
$$


Using $y_{1}(k)$ and $y_{2}(k)$, we can compute some observation values of $R_{12}(\tau)$. Denote by $\Delta=\Delta_{2}-\Delta_{1}$ the relative time delay between $x_{1}(k)$ and $x_{2}(k)$, there is

$$
\begin{aligned}
& \Re_{12}(n)=\frac{1}{N} \sum_{k=1}^{N} y_{1}(k) y_{2}(k+n) \\
& \approx E\left\{s_{1}(t) s_{2}(t+n T-\Delta)\right\}=R_{12}(n T-\Delta)
\end{aligned}
$$

where $N$ is the length of the samples used in calculation. The accuracy of $\Re_{12}(n)$ is affected by the level of measurement noises $v_{i}$ and the sample length $N$.

A widely used method to estimate $\Delta$ is to find the peak position of $\mathfrak{R}_{12}(n)$. Suppose that $\mathfrak{R}_{12}(n)$ takes on its maximum value at $n_{0}$. If $\Delta$ is an integral multiple of $T$, we simply have $\Delta=n_{0} T$. When $\Delta$ is a fractional multiple of $T$, the current technique is to fit a parabola function with points $\mathfrak{R}_{12}\left(n_{0}-1\right), \mathfrak{R}_{12}\left(n_{0}\right)$ and $\mathfrak{R}_{12}\left(n_{0}+1\right)$, and estimate $\Delta$ to be the peak position of the fitted parabola [4-5]. This kind of technique is simple but efficient and can estimate $\Delta$ to the desired precision. But two fundamental problems remain open: what is the exact expression of CCF $R_{12}(\tau)$ given some prior knowledge of ACF $R_{s}(\tau)$ and sensor kernels, and what is the best model to be used to fit $R_{12}(\tau)$ by the available observations $\Re_{12}(n)$ given a constraint on the model complexity? These are the issues to be addressed in the next sections.

\section{ANALYSIS OF CCF}

In this paper we consider the class of processes whose ACF can be written as an exponential function:

$$
R_{s}(\tau)=\alpha \cdot e^{-\beta|\tau|}
$$

where parameters $\alpha$ and $\beta$ are positive real numbers. This class of processes can represent many information sources such as the Gaussian Markov processes. Doob [7] showed that the ACF of any random Gaussian and Markov process can be modeled as an exponential function. Given $R_{s}(\tau)$, the CCF $R_{12}(\tau)$ depends only on the sensor kernel $g_{i}(t)$. Due to the physical limitation of sensors, in real systems $g_{i}(t)$ are low-pass filters. Next we analyze $R_{12}(\tau)$ for two important cases of sensor kernels used in many applications: Gaussian kernels and Box kernels.

\section{A. Gaussian kernels}

Suppose $g_{i}(t)$ is a Gaussian function centered at origin and has standard deviation $v_{i}$. Without loss of generality, we set $T=1$. To ensure that $g_{i}(t)$ is nearly zero outside sampling interval $[-1 / 2,1 / 2]$, we let $v_{i} \leq 1 / 4$. (But the following development is independent of this condition). Since kernels $g_{1}(t)$ and $g_{2}(t)$ are Gaussian, their convolution is also Gaussian. Let

$$
G(t)=g_{1}(-t) * g_{2}(t)=\frac{1}{\sqrt{2 \pi v}} e^{-t^{2} /\left(2 v^{2}\right)}
$$

where $v=\sqrt{v_{1}^{2}+v_{2}^{2}}$ is the standard deviation of $G(t)$. For all $v_{1}, v_{2} \leq 1 / 4$, we have $v \leq \sqrt{2} / 4$. After some computation, we can derive that

$$
R_{12}(\tau)=R_{s}(\tau) * G(\tau)=C \cdot\left(f_{l}(\tau)+f_{r}(\tau)\right)
$$

where constant $C=\frac{\alpha}{2} e^{v^{2} \beta^{2} / 2}$ is independent of $\tau$ and

$$
f_{l}(\tau)=e^{\tau \beta} \operatorname{erfc}\left(\frac{v^{2} \beta+\tau}{\sqrt{2} v}\right), f_{r}(\tau)=e^{-\tau \beta} \operatorname{erfc}\left(\frac{v^{2} \beta-\tau}{\sqrt{2} v}\right)
$$

where $\operatorname{erfc}(x)=\frac{2}{\sqrt{\pi}} \int_{x}^{\infty} e^{-t^{2}} d t$ is the complementary error function.

The shape of $R_{12}(\tau)$ is determined by $f(\tau)=f_{l}(\tau)+f_{r}(\tau)$. Since $f(\tau)$ is an even function, it suffices to discuss the case when $\tau \geq 0$. Note that the decreasing speed of $\operatorname{erfc}\left(\left(v^{2} \beta+\tau\right) / \sqrt{2} v\right)$ is faster than the increasing speed of $e^{\tau \beta}$. So when $\tau$ is greater than some positive number, $f_{l}(\tau) \rightarrow 0$ and $\operatorname{erfc}\left(\left(v^{2} \beta-\tau\right) / \sqrt{2} v\right) \rightarrow 2$ and then $R_{12}(\tau) \approx C \cdot f_{r}(\tau) \rightarrow 2 C \cdot e^{-\tau \beta}$. That is to say, when $|\tau|$ is large, $f(\tau)$ and in turn $R_{12}(\tau)$ can be approximately modeled by an exponential function.

However, in time delay estimation by locating the peak position of $R_{12}(\tau)$ we are more interested in the behavior of $R_{12}(\tau)$ around origin. The shape of $R_{12}(\tau)$ depends on two parameters, $\beta$, the decaying parameter of ACF $R_{s}(\tau)$, and $v$, the standard deviation of $G(x)$ in (5). When $v$ decreases, $G(x)$ shapes more like a Dirac function, hence the shape of $R_{12}(\tau)$ approaches to that of $R_{s}(\tau)$ due to convolution $R_{12}(\tau)=R_{s}(\tau) * G(\tau)$. Similarly, when $v$ is fixed and $\beta$ increases, the shape of $R_{s}(\tau)$ approaches to a Dirac function and then the shape of $R_{12}(\tau)$ approaches to the Gaussian function $G(x)$.

\section{B. Box kernels}

Another common sensor kernel is the Box function, i.e., $g_{i}(t)$ is constant in support $[-1 / 2,1 / 2]$. In other words, the sensor samples the observed process as an integrator in each sampling period. Since that $g_{1}(t)$ and $g_{2}(t)$ are Box kernels, their convolution is

$$
G(t)=g_{1}(-t) * g_{2}(t)= \begin{cases}1+t & -1 \leq t \leq 0 \\ 1-t & 0 \leq t \leq 1 \\ 0 & |t|>1\end{cases}
$$

Because CCF $R_{12}(\tau)=R_{s}(\tau) * G(\tau)$ is an even function, we only consider the case for $\tau \geq 0$.

After some tedious computations, we have 


$$
\begin{gathered}
R_{12}(\tau)=\alpha\left(2 \frac{1-\tau}{\beta}+\frac{1}{\beta^{2}} e^{-\beta} e^{\beta \tau}+\frac{1}{\beta^{2}}\left(e^{-\beta}-2\right) e^{-\beta \tau}\right), \\
\quad 0 \leq \tau \leq 1 \\
R_{12}(\tau)=\frac{\alpha}{\beta^{2}}\left(e^{\beta}+e^{-\beta}-2\right) e^{-\beta \tau}, \tau>1
\end{gathered}
$$$$
0 \leq \tau \leq 1 \quad(9-\mathrm{a})
$$

When $|\tau|>1, R_{12}(\tau)$ is an exponential function, and when $|\tau| \leq 1, R_{12}(\tau)$ is a linear combination of exponential terms $e^{-\beta \tau}$ and $e^{\beta \tau}$ and linear term $\tau$. The shape of $R_{12}(\tau)$ is controlled by $\beta$. (Parameter $\alpha$ only affects the magnitude scale of $R_{12}(\tau)$.) With the increasing of $\beta$, the shape of ACF $R_{s}(\tau)$ approaches to a Dirac function so that the shape of CCF $R_{12}(\tau)$ approaches to that of $G(t)$ defined in $(8)$, which is actually the first order spline function.

\section{TIME DELAY ESTIMATION BY CCF FITTING}

Unfortunately, $\mathrm{CCF} R_{12}(\tau)$ does not have a simple closed form, as revealed in the proceeding section. But $R_{12}(\tau)$ is a smooth even function and it exhibits a quadratic-like behavior near the origin. This explains the past success of parabola fitting of $R_{12}(\tau)$ near the peak position. Suppose that $\Re_{12}(n)$ are the true samples of $R_{12}(\tau)$, i.e., $\Re_{12}(n)=R_{12}(n T-\Delta)$. The task of time delay estimation is to determine $\Delta$ from the parametric model fitted by points $\Re_{12}\left(n_{0}-1\right), \Re_{12}\left(n_{0}\right)$ and $\Re_{12}\left(n_{0}+1\right)$. If $\Delta$ is an integral multiple of sampling period $T$, we have $\Delta=n_{0} T$. However, in practice $\Delta$ has an arbitrary real value and the fractional part of $\Delta$ can be written as

$$
\Delta_{\varepsilon}=\Delta-n_{0} T
$$

Denote parabola $f_{p}(x)=a x^{2}+b x+c$, whose parameters are determined by the three significant samples. The fractional part $\Delta_{\varepsilon}$ is estimated as

$$
\hat{\Delta}_{p}=-b T / 2 a
$$

A natural question is if there exists a better model of $R_{12}(\tau)$ in the neighborhood of origin given the same number of model parameters? Intuitively the Gaussian function $f_{g}(x)=a \cdot e^{-b(x-c)^{2}}$ is a good candidate. Let $\Re_{12}\left(n_{0}-1\right)=f_{g}(-1), \quad \Re_{12}\left(n_{0}\right)=f_{g}(0) \quad$ and $\Re_{12}\left(n_{0}+1\right)=f_{g}(1)$, we have

$$
c=\frac{\ln \Re_{12}\left(n_{0}+1\right)-\ln \Re_{12}\left(n_{0}-1\right)}{4 \ln \Re_{12}\left(n_{0}\right)-2 \ln \Re_{12}\left(n_{0}-1\right)-2 \ln \Re_{12}\left(n_{0}+1\right)}
$$

and the fractional part $\Delta_{\varepsilon}$ is estimated as

$$
\hat{\Delta}_{g}=c T
$$

Denote by $D_{p}=\hat{\Delta}_{p}-\Delta_{\varepsilon}$ and $D_{g}=\hat{\Delta}_{g}-\Delta_{\varepsilon}$ the estimation errors of the two different fitting models, next we compare the magnitudes of them via numerical computations.

\section{A. Results for Gaussian sensor kernels}

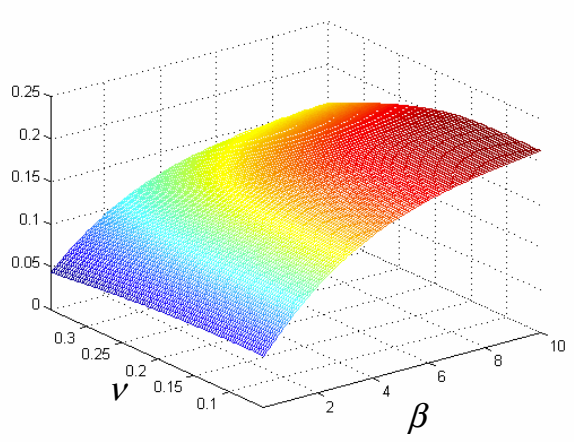

(a)

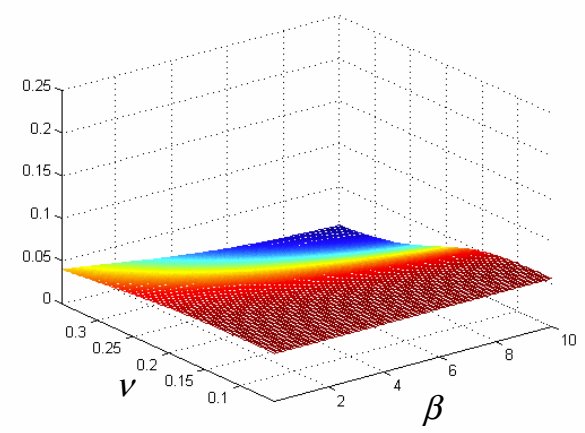

(b)

Figure 2. Surfaces (a) $E_{p}$ and (b) $E_{g}$ vs. parameters $v$ and $\beta$.

When sensor kernels are Gaussian, the CCF $R_{12}(\tau)$ is determined by (6) and its shape is controlled by $\beta$ and $v$. Normalize $T=1$ so that the fractional part of $\Delta$ is $\Delta_{\varepsilon} \in[-0.5,0.5]$. We increase $\Delta_{\varepsilon}$ from -0.5 to 0.5 , and sample $\Re_{12}\left(n_{0}-1\right), \Re_{12}\left(n_{0}\right)$ and $\Re_{12}\left(n_{0}+1\right)$ from $R_{12}(\tau)$, then we compute the two estimates of $\Delta_{\varepsilon}, \hat{\Delta}_{p}$ and $\hat{\Delta}_{g}$, and their estimation errors $D_{p}$ and $D_{g}$. Define the mean absolute errors (MAS) of $\hat{\Delta}_{p}$ and $\hat{\Delta}_{g}$ as

$$
E_{p}=\int_{0.5}^{0.5}\left|D_{p}\left(\Delta_{\varepsilon}\right)\right| d \Delta_{\varepsilon} \text { and } E_{g}=\int_{-.5}^{0.5}\left|D_{g}\left(\Delta_{\varepsilon}\right)\right| d \Delta_{\varepsilon}
$$

Fig. 2 plots $E_{p}$ and $E_{g}$ as two-dimensional functions over $v \in[0.05,0.35]$ and $\beta \in(0,10]$. Clearly, the estimation error $E_{p}$ is greater than $E_{g}$ in the entire range of $(\nu, \beta)$. Both $E_{p}$ and $E_{g}$ decrease in $v$. However, with the increasing of $\beta, E_{p}$ increases rapidly but $E_{g}$ decreases. These observations can be explained as follows. First, a smaller $v$ makes $G(t)$ in (5) sharper and closer to a Dirac function, so that the shape of $R_{12}(\tau)$ is more like that of 
$R_{s}(\tau)$, which is an exponential function, due to the convolution $R_{12}(\tau)=R_{s}(\tau) * G(\tau)$. Thus the fitting error between $R_{12}(\tau)$ and parabola/Gaussian function increases in $v$, so does the MAS $E_{p}$ or $E_{g}$. Second, a larger $\beta$ drives $R_{s}(\tau)$ closer to a Dirac function, so that that the shape of $R_{12}(\tau)$ approaches that of $G(t)$, which is a Gaussian function. Therefore, the fitting error between $R_{12}(\tau)$ and Gaussian function decreases in $\beta$, whereas the fitting error between $R_{12}(\tau)$ and parabola function increases. This is the reason why MAS $E_{p}$ and $E_{g}$ have opposite trends in $\beta$. Finally, as shown in Fig. 2, the Gaussian model of the CCF $R_{12}(\tau)$ is more robust than the parabola one in time delay estimation.

\section{B. Results for Box sensor kernels}

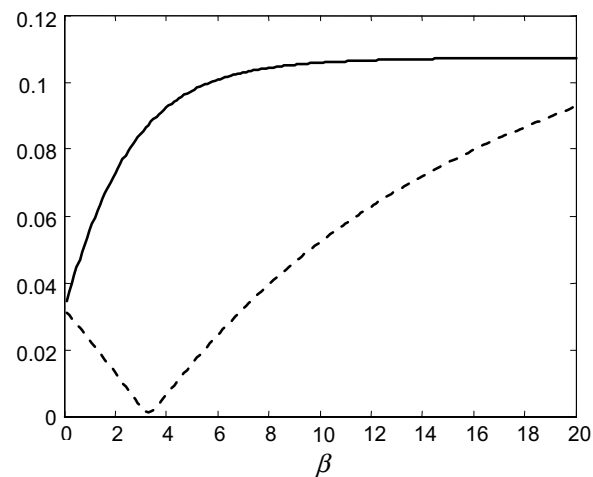

Figure 3. The curves of $E_{p}$ (solid) and $E_{g}$ (dashed) versus parameter $\beta$.

The CCF $R_{12}(\tau)$ for Box kernels is given by (9) and its shape is controlled by $\beta$ only. Fig. 3 plots the curves of $E_{p}$ and $E_{g}$ versus $\beta$ in interval $(0,20]$. We see that $E_{p}$ increases in $\beta$ and it flattens out when $\beta>10$. In a wide range of $\beta, E_{g}$ is much less than $E_{p}$, and $E_{g}$ becomes greater than $E_{p}$ only when $\beta>26$. In real applications $\beta$ is usually small, rarely greater than 10 . A large value of $\beta$ means the considered process is nearly a white process whose ACF is a Dirac pulse. $E_{g}$ reaches its minimum around $\beta=3.3$ where $E_{g}$ is almost zero and $R_{12}(\tau)$ can be perfectly approximated by a Gaussian function. As $\beta$ gets larger, the ACF $R_{s}(\tau)$ becomes closer to a Dirac function so that the shape of CCF $R_{12}(\tau)$ approaches to that of the spline function $G(t)$ in (8). Consequently the fitting error between $R_{12}(\tau)$ and the Gaussian function, as well as the MAS $E_{g}$, increases in $\beta$.

\section{CONCLUSION}

Analytic forms of cross correlation functions (CCF) were derived and their behaviors were examined for stationary processes whose auto-correlation function is exponential and for two important types of sensor sampling kernels: Gaussian and Box. Under these conditions, a Gaussian approximation model of CCF was proposed and shown to be more accurate and robust than the current parabola-based CCF model in time delay estimation. In these analyses, we supposed that $\mathfrak{R}_{12}(n)$ are accurate samples of $R_{12}(\tau)$. In practice, $\Re_{12}(n)$ are nonideal observations of $R_{12}(\tau)$ and the accuracy subjects to the level of measurement noises $v_{i}$ and the sample length $N$. We also performed simulations to test the performances of the proposed method on different noise level and sample length. (Because of the limitation of space, we can not put the detail simulation results in this paper.) The results also showed that the Gaussian model is more accurate and robust than the parabola CCF model.

\section{REFERENCES}

[1] C. H. Knapp and G. C. Carter, "The generalized correlation method for estimation of time delay," IEEE Trans. Acoustics, Speech, and Signal Processing, vol. ASSP-24, pp. 320-327, Aug. 1976.

[2] M. Azaria and D. Hertz, "Time delay estimation by generalized cross correlation methods," IEEE Trans. Acoustics, Speech, and Signal Processing, vol. ASSP-32, pp. 280-285, June 1984.

[3] G. C. Carter, "Coherence and time delay estimation," Proceedings of the IEEE, vol. 75, pp. 236-255, Feb. 1987.

[4] R. Moddemeijer, "On the determination of the position of extrema of sampled correlators," IEEE Trans. Signal Processing, vol. 39, pp. 216-219, Jan. 1990.

[5] G. Jacovitti and G. Scarano, "Discrete time techniques for time delay estimation," IEEE Trans. Signal Processing, vol. 41, pp. 525-533, Feb. 1993.

[6] A. Kumar and Y. Bar-Shalom, "Time-domain analysis of cross correlation for time delay estimation with an autocorrelated signal," IEEE Trans. Signal Processing, vol. 41, pp. 1664-1668, April 1993.

[7] J. L. Doob, "Topics in the theory of Markov chains," Trans. Amer. Math. Soc., vol. 52, pp. 37-64, 1942.

[8] A. Papoulis, Probability, Random Variables, and Stochastic Processes, McGraw-Hill High Education, $3^{\text {rd }}$ edition, 1991. 